When compared with rates in similar State Hospitals in the 1970s tardive dyskinesia was now half the rate and Parkinsonism about the same.

Conclusion. Overall rates of movement disorder are not very different between FGA and SGA. The surprise was that intention tremor only occurred with SGAs. Why?

\section{The effect of ketamine on psychopathology and implications for understanding schizophrenia and its therapeutic use: a meta-analysis}

Katherine Beck ${ }^{1 \star}$, Guy Hindley ${ }^{1}$, Faith Borgan ${ }^{1}$, Cedric Ginestet ${ }^{1}$, Robert McCutcheon ${ }^{1}$, Stefan Brugger ${ }^{1}$, Naomi Driesen ${ }^{2}$, Mohini Ranganathan ${ }^{2}$, Deepak D'Souza ${ }^{2}$, Matthew Taylor ${ }^{3}$, John Krystal ${ }^{2}$ and Oliver Howes ${ }^{1}$

${ }^{1}$ IoPPN King's College London; ${ }^{2}$ Yale University School, Dept. of Psychiatry and National Centre for PTSD, VA Connecticut and

${ }^{3}$ University Department of Psychiatry, Warneford Hospital ${ }^{\star}$ Corresponding author.

doi: 10.1192/bjo.2021.634

Aims. To conduct a meta-analysis of the effect of ketamine on psychopathology in healthy volunteers and patients with schizophrenia, and the experimental factors affecting this.

Background. Ketamine is increasingly used to treat depression and other psychiatric disorders but can induce schizophrenia-like symptoms. Despite this, the consistency and magnitude of symptoms induced by ketamine, or what factors influence the effects of ketamine on these remain unknown.

Method. MEDLINE, EMBASE and PsychINFO databases were searched for within-subject placebo controlled studies reporting symptoms using the Brief Psychiatric Rating Scale (BPRS) or Positive and Negative Syndrome Scale (PANSS) in response to an acute ketamine challenge in healthy participants or people with schizophrenia. Two independent investigators extracted study-level data for a random-effects meta-analysis. Total, positive and negative BPRS and PANSS scores were extracted. Sub-group analyses were conducted examining the effect of: blinding status, ketamine preparation, infusion method and time between ketamine and placebo condition. Standardized mean change scores were used as effect sizes for individual studies. Standardized mean changes between ketamine and placebo for total, positive and negative BPRS and PANSS were calculated.

Result. Of 7819 citations retrieved, 36 studies involving healthy participants were included. The overall sample included 725 healthy volunteers exposed to both the ketamine and placebo condition. Ketamine induced a significant increase in transient psychopathology in healthy participants, for total (Standardized mean change $(\mathrm{SMC})=1.50(95 \% \mathrm{CI}=1.23$ to 1.77$), \mathrm{p}<0.0001)$, positive $(\mathrm{SMC}=$ $1.55(95 \% \mathrm{CI}=1.29$ to 1.81$), \mathrm{p}<0.0001)$ and negative $(\mathrm{SMC}=1.16$, (95\% $\mathrm{CI}=0.96$ to 1.35$), \mathrm{p}<0.0001$ ) symptom ratings, relative to the placebo condition. This effect was significantly greater for positive symptoms than negative symptoms $(p=0.004)$. Bolus followed by constant infusion increased ketamine's effect on positive symptoms relative to infusion alone $(\mathrm{p}=0.006)$. Single-day study design increased ketamine's effect on total symptoms $(p=0.007)$, but age and gender did not moderate effects. There were insufficient studies for meta-analysis of studies in schizophrenia. Of these studies, two found a significant increase in symptoms with ketamine administration in total and positive symptoms. Only one study found an increase in negative symptom severity with ketamine.

Conclusion. These findings show that acute ketamine administration induces schizophrenia-like symptomatology with large effect sizes but there is a greater increase in positive than negative symptoms, and when a bolus is used. These findings suggest bolus doses should be avoided in its therapeutic use to minimize the risk of inducing transient positive psychotic symptoms.

\section{Evaluation of self-esteem in adolescents of secondary school level}

Farhana Begum ${ }^{1 \star}$, Usama Zubair ${ }^{2}$, Iqbal Afridi ${ }^{3}$, Fatima Toufique ${ }^{4}$, Jawed Dars ${ }^{3}$ and Chunni Lal ${ }^{3}$

${ }^{1}$ Patel Hospital; ${ }^{2}$ Conolly Hospital; ${ }^{3}$ Jinnah Postgraduate Medical Centre and ${ }^{4}$ Sindh Rangers Hospital

${ }^{\star}$ Corresponding author.

doi: 10.1192/bjo.2021.635

Aims. To assess the frequency of low self-esteem among adolescents of secondary school level in private schools of Karachi Method. It was a cross-sectional descriptive study done in ten private schools of Karachi for a period of 6 months. The proposal of the study was accepted by ethical committee of Jinnah Postgraduate Medical Centre, Karachi (called Institutional Review Board or IRB).The subjects fulfilling inclusion criteria were enrolled after obtaining informed consent by their parents. A semi-structured Performa was used to assess students' particulars and included Rosenberg Self-esteem Questionnaire as a part of Performa. The data were analysed using SPSS version 17.0. Frequencies \& percentages were generated for the level of self-esteem.Stratified analysis was done with a $\mathrm{p}$ value $<0.05$ taken as significant.

Result. Out of the 246 students, $39.8 \%$ were of 14 yrs of age, $36.2 \%$ were 15 years of age, while only $24 \%$ of adolescents were 16 years of age. Majority $(64.2 \%)$ of the students were males while females were $35.8 \% .70 .3 \%$ of the students had normal level of self-esteem, whereas $28.9 \%$ of students had low self-esteem and only $0.8 \%$ of students had high self-esteem. Relationship of all the variables was found to be non-significant except that of educational level ( $p$-value 0.047). Conclusion. Self-esteem was found to be normal in most of the adolescents of secondary school level but still more than $1 / 4$ th of the study participants had low self-esteem which if assessed and addressed early may save the individuals from mental health issues as well as problems at work and home life.Having a better understanding of self-esteem, can help us to identify the adolescents who have low self-esteem and are predisposed to develop mental health difficulties in future.It can lead to not only early intervention and reducing the burden of disease but also help in developing programs to help improve self-esteem in adolescents,hence increasing their overall motivation and productivity.

\section{Mental health, physical impairment and violence} among FSWS in North Karnataka, South India: a story of intersecting vulnerabilities

Alicja Beksinska ${ }^{1 \star}$, Tara S Beattie ${ }^{1}$, Lucy Platt ${ }^{2}$, Parinita Bhattacharjee ${ }^{3}$, Ravi Prakash ${ }^{3}$, Satyanarayana Ramanaik ${ }^{4}$, Kavitha Dibbadahalli ${ }^{3}$, Martine Collumbien ${ }^{1}$, Mitzy Gafos ${ }^{1}$, Calum Davey ${ }^{2}$, Charlotte Watts ${ }^{1}$, Shajy Isac ${ }^{4}$ and Rachel Jewkes ${ }^{5}$

${ }^{1}$ Department of Global Health and Development, London School of Hygiene and Tropical Medicine; ${ }^{2}$ Department of Public Health, Environments and Society, London School of Hygiene and Tropical Medicine; ${ }^{3}$ Karnataka Health Promotion Trust; ${ }^{4}$ Karnataka Health Promotion Trust, University of Manitoba, Department of Community Health Sciences and ${ }^{5}$ Gender and Health Division, South African Medical Research Council

${ }^{\star}$ Corresponding author.

doi: 10.1192/bjo.2021.636 